\title{
Research on the Mechanism of Pulsatilla Potentially Useful for the Treatment of Triple Negative Breast Cancer Based on Network Pharmacology
}

\section{Shilin Li ( $\square$ lishilinty@163.com )}

Chinese Academy of Medical Sciences Cancer Institute and Hospital: Cancer Hospital Chinese Academy of Medical Sciences

\section{Yi Fang}

Chinese Academy of Medical Sciences Cancer Institute and Hospital: Cancer Hospital Chinese Academy of Medical Sciences

\section{Research Article}

Keywords: Triple negative breast cancer, Pulsatilla Chinensis, Caspase 3

Posted Date: May 17th, 2021

DOI: https://doi.org/10.21203/rs.3.rs-516623/v1

License: (c) (i) This work is licensed under a Creative Commons Attribution 4.0 International License. Read Full License 


\section{Abstract}

Triple negative breast cancer is a very malignant type of breast cancer. Its age of onset is young and the prognosis is poor, which seriously threatens women's physical and mental health. Pulsatilla Chinensis is a common medicine in traditional Chinese medicine. Application of network pharmacology analysis found that the active ingredients in Pulsatilla can target Caspase-3, NOS3, etc. to exert anti-tumor effects. This discovery will provide new alternative drugs and potential methods for the treatment of triplenegative breast cancer.

\section{Introduction}

Breast cancer is a highly heterogeneous tumor disease which seriously endangers women's physical and mental health. Although with the continuous progress and development of science and technology and society, the spectrum of human cancer is also quietly changing, breast cancer still occupies the second leading killer of women. According to estimates by Globocan 2020 [1], there are about 2.26 million new cases of female breast cancer worldwide, and about 680,000 deaths and the incidence of breast cancer is gradually becoming younger. Among female cancers in our country, breast cancer is likewise the most common, with more than 400,000 new cases during 2020 [1]. Although the incidence of breast cancer in developed countries has declined to a certain extent, the incidence of breast cancer in underdeveloped countries and regions is still increasing [2.3], and people should pay sufficient attention. It is currently believed that the incidence of breast cancer may be linked to hormones, family history, early menarche, late menopause, late birth, high-fat diet, and overnutrition, but the exact cause has not been clarified. The molecular classification of breast cancer mainly includes LuminalA, LuminalB, triple-negative, and HER-2 positive. Triple negative breast cancer (TNBC) is highly malignant, invasive, and prone to metastasis. $10 \%-15 \%$ of breast cancers occur [4]. Triple negative breast cancer refers to breast cancer with negative expression of estrogen receptor (ER), progesterone receptor (PR), and human epidermal growth factor receptor 2 (HER2). Due to its high degree of heterogeneity, aggressiveness and lack of treatment options, TNBC has become the deadliest breast cancer subtype. Lack of effective treatment options, endocrine therapy and targeted therapy is not satisfactory. TNBC is more common among young patients. The tumor is usually larger, poorly differentiated, more likely to show lymph node involvement at the time of diagnosis, and more biologically aggressive [5]. Chemotherapy is the main treatment for TNBC, taxanes, and tetracycline are the main treatment drugs, but patients are prone to chemotherapy resistance after treatment; even if the patient receives the standard systemic chemotherapy, the 5-year survival of patients with metastatic TNBC rate is still below $30 \%$. The single-agent treatment plan is set to multi-drug combination chemotherapy to increase the remission rate, which can increase the chemotherapy effect to a certain extent, but this also increases the probability of chemotherapy's toxicity [4]. Therefore, finding new therapeutic drugs and methods to relieve chemotherapy resistance has become an urgent problem to be resolved.

Natural compounds refer to biological products derived from animals, plants, and microorganisms. They have been long since clinical use, are a rich source of biologically active compounds, and are ideal for 
screening biologically active small molecules. Natural products have high value in the research and development of anti-cancer drugs, and many drug development projects are continuing [6]. For example, in the field of anti-cancer drugs, from the 1940s to the end of 2014, 131 (or 75\%) of the 175 small molecules approved by the US FDA were not "S" (synthetic) molecules, of which 85 ( or 49\%), actually natural products or directly extracted from natural products [7]. It can be seen that natural products still have great potential in the field of anticancer, and they are a potential treasure house for the development of novel anticancer drugs and resistance to chemotherapy.

Pulsatilla Chinensis, a classic and commonly used Chinese medicine, aliases as Nahograss, Pulsatilla, etc, is a natural compound. The medicinal part is usually the dry root of Pulsatilla, a plant of the Ranunculaceae family, widely distributed in Jilin, Liaoning, Hebei, and Shandong of China, Henan, Shanxi, Shaanxi, Heilongjiang, and other provinces of hills, barren slopes, and fields. Sexual bitterness, coldness, returns to stomach, large intestine meridian, mainly used for clearing heat and detoxification, cooling blood to stop dysentery. In recent years, Pulsatilla has been reported to have definite anti-cancer activity in a variety of cancers. For example, experiments have found that Pulsatilla can induce apoptosis of colon cancer cells by acting on TP53, Caspase-9, and BCL-2 [8], and similarly, it can also promote apoptosis of lung cancer cells [9]; in liver cancer and pancreas Studies in mouse models of cancer have found that Pulsatilla can induce cancer cells' death and inhibit tumor's growth [10]; Pulsatilla has also been reported to inhibit cancer cells' proliferation, invasion, and metastasis in breast cancer [11]. Based on the excellent anti-cancer properties of Pulsatilla and the current status that there is no ideal therapeutic drug for triple negative breast cancer, this article intends to adopt a series of network pharmacology research methods, according to the chemical composition of Pulsatilla, the target of chemical composition, and the related genes of triple negative breast cancer. Protein interaction and other information, construct protein interaction network, and analysis, and strive to reveal the complex mechanism of Pulsatilla as a potential drug to treat triple-negative breast cancer with multiple components, multiple targets, and multiple pathways, and make preliminary verifications. To provide a basis for further basic experimental research and provide new ideas and directions for the development of new drugs for the treatment of triple-negative breast cancer.

\section{Materials And Methods}

\subsection{Pulsatilla ingredients and targets}

The chemical composition of Pulsatilla was retrieved through Traditional Chinese Medicine Systems Pharmacology(TCMP) [12] and a total of 57 chemical compositions were retrieved. After screening according to bioavailability $(\mathrm{OB})>=30 \%$ and drug-like properties $>=0.18,11$ chemical compositions were obtained. Search the target points of the relevant chemical components in TCMSP, delete the components without target information, and get a total of 9 effective chemical components finally. A total of 138 targets were found in 9 corresponding active ingredients.

\subsection{Triple negative breast cancer related genes}


Searching for triple negative breast cancer related gene targets on the DisGeNet website, a total of 1598 results were obtained, and screening was carried out according to the Score_gda $>=0.05$ standard, and 133 related gene targets were finally obtained.There are 11 same genes between the active component targets of Pulsatilla and the triple-negative breast cancer targets

\subsection{Protein-protein interaction information}

Search for protein interaction information through STRING (version: 11.0), and set the search condition species of "Homo Sapiens".

\subsection{Network Construction}

Construct a "compound-compound target network" by connecting the effective chemical components of Pulsatilla and its related targets; construct a "compound target protein interaction network" by connecting related targets and the proteins that interact with them; Negative breast cancer related gene targets and proteins that interact with them, construct a "triple negative breast cancer related target protein interaction network". Take the intersection of "compound target protein interaction network" and "triple negative breast cancer related target protein Interaction network" to construct the "compound target-triple negative breast cancer-related target protein interaction network" through Cytoscape (version: 3.4.2) for network visualization.

\subsection{Hub gene analysis}

"Compound Target-Triple Negative Breast Cancer Related Target Protein Interaction Network" was analyzed by Cytohubba, a plug-in Cytoscape. Twelve methods such as MCC, Degree, MNC and so on were used to calculate, and a total of 26 possible key genes were counted.

\subsection{GO enrichment analyses}

Use DAVID (version: 6.8) website to perform GO enrichment analysis and map the intersection genes between compound component targets and triple negative breast cancer targets.

\subsection{KEGG pathway enrichment analysis}

KEGG enrichment analysis was conducted on the intersection genes (a total of 11) between the compound component target and the triple negative breast cancer target. As a result, a total of 25 pathways were obtained and a bubble chart was made. Most genes are focused on cancer related pathways (hsa05200).

\subsection{Drugs}

Beta-Sitosterol (5mg,purity:99.95\%) was purchased from Topscience Corporate (Shanghai,China), dissolved in DMSO to a concentration of $10 \mathrm{mM}$, stored at $-80^{\square} \mathrm{C}$. 


\subsection{Cell culture}

MDA-MB-231 cells were purchased from ATCC and grown in L-15 supplemented with $10 \%$ FBS in a saturated humidity incubator $\left(37^{\square} \mathrm{C} \otimes 0 \% \mathrm{CO}_{2}\right)$.

\subsection{CCK-8 assay}

Cell viability was determined using a CCK-8 assay according to the manufacturer's protocol. MDA-MB-231 cells were seeded at a density of $1.2 \times 10^{4}$ cells/well in a 96-well culture plate for $24 \mathrm{~h}$ after which the medium was changed and Beta-Sitosterol $(0,50,100 \mu \mathrm{M} / \mathrm{mL})$ was added and cultured for a further for 24 , 48 or $72 \mathrm{~h} .10 \mu \mathrm{l} \mathrm{CCK}-8$ reagent was added to each well and incubated for $1 \mathrm{~h}$. The optical density at 450 nm was measured using a Veriti 96-well Thermal Cycler (Applied Biosystem).

\subsection{Western blot analysis}

Proteins were extracted from MDA-MB-231 cells using RIPA buffer (Yeasen Biotech Co., Ltd.) and the concentration was measured using a bicinchoninic acid assay. A total of $20 \mu \mathrm{g}$ protein was loaded into each lane of a $10 \%$ polyacrylamide gel and separated by SDS-PAGE. After the proteins were resolved, they were transferred to a PVDF membrane (EMD Millipore, Billerica, MA, USA). Membranes were blocked with $5 \%$ non-fat dried milk, incubated with anti-Caspase 3 (1:1000), anti-beta-Tubulin (1:1000), antibodies overnight at $4{ }^{\circ} \mathrm{C}$, and then incubated with the horseradish peroxidaseconjugated secondary antibodies (1:4000) for $2 \mathrm{~h}$ at room temperature. SuperSignal ${ }^{\mathrm{TM}}$ West Femto Maximum (Thermo Fisher Scientific) and SuperSignal ${ }^{\mathrm{TM}}$ West Pico PLUS (Thermo Fisher Scientific) kits were used to visualize bands Besides, beta-Tubulin was used to quantify the amount and integrity of the proteins.

\section{Results}

\subsection{Compound-compound target network analysis}

In TCMSP, set bioavailability (OB) $>=30 \%$ and drug-likeness $>=0.18$ to screen and filter out the components without target information, and obtain 9 effective compound chemical components. The specific information is shown in Table 1. The compound-compound target is shown in Figure 1, which reflects the multi-targets potential of Pulsatilla in the treatment of triple negative breast cancer. At the same time, most targets are connected to multiple chemical components, which reflect the role of multiple components in the treatment of cancer. For example, $\mathrm{PgR}$ (progesterone receptor) is an important molecular phenotype in breast cancer. It can be used as one of the ]basis for breast cancer classification. At the same time, its expression can help predict whether the patient is sensitive to endocrine therapy [13]. Important clinical significance. Multiple active ingredients in Pulsatilla simultaneously target PgR, suggesting that PgR may be used as one of the targets of Pulsatilla in the treatment of breast cancer.

\subsection{Compound target-triple negative breast cancer related target protein interaction network and key} genes 
The intersection of compound component targets and triple negative breast cancer targets are 11 genes(Figure 2a). By connecting the compound-related targets and the proteins that interact with them, a "compound target protein interaction network" is constructed(Figure 2b); by connecting the triple-negative breast cancer-related gene targets and the proteins that interact with them, a "triple-negative breast cancer-related Target protein interaction network" is constructed (Figure 2b). To further explore the potential pharmacological mechanism of Pulsatilla in the treatment of triple-negative breast cancer, the "compound target protein interaction network" and the "triple negative breast cancer related target protein interaction network" were intersected to construct a "compound target-triple negative breast cancer related target protein interaction network"(Figure 2b). Network analysis shows that the network consists of 205 nodes and 3206 edges. Subsequently, using Cytoscape plug-in cytohubba's 12 calculation methods MCC, Degree, MNC, etc., the network was analyzed to screen out the hub gene. There are 32 genes in the screening results, of which caspase-3 appears as hub genes in 11 calculation methods, and the frequencies of other genes appearing in each calculation method are 6 times, 5 times, 4 times, and 1 time respectively(Figure 2c). Thus, Caspase-3 is most likely to be a potential target of Pulsatilla in the treatment of triple negative breast cancer. Caspase- 3 plays a very important role in apoptosis. Caspase is a widely expressed intracellular cysteine protease that mediates cell death and inflammation, while caspase-3 mainly regulates the death process of apoptosis and necrotic cells [14]. As an effector protein downstream of apoptosis, caspase-3 can inactivate and lyse proteins related to important cell functions, such as cytoskeleton maintenance, cell cycle regulation, DNA repair, signal transduction, etc. [15].

\subsection{GO enrichment analysis results}

According to GO enrichment analysis, the compound component targets and triple negative breast cancer targets overlap genes' (a total of 11) biological processes are mainly concentrated on the downregulation of gene expression, cell components are mainly concentrated in the nucleus, and molecular functions are mainly enriched in enzyme binding(Figure 3a).

\subsection{KEGG pathway enrichment analysis}

By the KEGG enrichment analysis, the following bubble chart can be obtained (Figure 3b). We found that genes are enriched in cancer pathways, apoptosis pathways, estrogen signaling pathways, etc. As shown in Figure 4, the cancer pathway contains the largest number of potential targets for Pulsatilla, which should be confirmed as the potential of Pulsatilla for the treatment of triple-negative breast cancer. Core target pathway. Cancer-related pathways include classic pathways such as ERK signaling pathway, PI3K signaling pathway, and WNT pathway. These pathways all play important roles in the occurrence and development of cancer.

\subsection{Experiments verify that the potential target of Pulsatilla in the treatment of triple-negative breast cancer may be Caspase-3}


Previous experimental studies [16] have proved that Beta-Sitosterol can affect the expression of caspase3 in the triple-negative breast cancer cell line MDA-MB-231.

For further verification, the active ingredient Beta-Sitosterol in Pulsatilla was used to treat breast cancer cell line MDA-MB-231 with different concentrations, and the proliferation curve of MDA-MB-231 was measured by the CCK8 method, and the expression of protein Caspase-3 in MDA-MB-231 treated with different concentrations of Beta-Sitosterol was determined by Western blotting.

As shown in Figure 4a, the experimental results found that the proliferation ability of MDA-MB-231 cells treated with Beta-Sitosterol was inhibited, and the inhibition intensity was time-dependent and concentration-dependent.

Western blot results showed(Figure 4b) that after treating MDA-MB-231 cells with Beta-Sitosterol, the expression of caspase-3 in MDA-MB-231 cells decreased, and the degree of decrease was concentrationdependent.

The experimental results confirmed the results of bioinformatics analysis. Caspase- 3 is most likely to be a potential target for Pulsatilla in the treatment of triple-negative breast cancer.

\section{Discussion}

In this study, the prediction and interpretation of the mechanism of Pulsatilla as a potential drug for the treatment of triple negative breast cancer was carried out by integrating network pharmacology methods such as target prediction and network construction. Firstly, the target prediction of 9 effective chemical components of Pulsatilla was performed, and 139 potential targets of Pulsatilla were obtained, which reflects the therapeutic characteristics of the synergistic effect of multi-component and multi-targets of traditional Chinese medicine. Secondly, through the comprehensive analysis among the "compound target-compound target network", "compound target protein interaction network", "triple negative breast cancer related target protein interaction network", and "compound target-triple negative breast cancer related target protein interaction network", it shows that caspase-3 may be the key target of Pulsatilla. Pulsatilla may also regulate proteins which related to cell proliferation and division, cell cycle, inflammation, etc., such as TP53, STAT3, MYC, NOS2, etc. which play the roles of anti-breast cancer through multiple channels. Finally, according to the results of KEGG pathway enrichment analysis, the core proteins regulated by Pulsatilla are mostly enriched in cancer-related pathways, estrogen-related pathways, apoptosis-related pathways and so on. These are closely related to the uncontrolled cell proliferation, cell invasion and metastasis of triple negative breast cancer.

In summary, this study uses network pharmacology methods to reveal the mechanism of Pulsatilla as a potential drug for the treatment of triple negative breast cancer, it may be related to the coordinated regulation of apoptosis, cell cycle, and cell proliferation. At the same time, this study provides new ideas and methods for the application of network pharmacology to explore the specific mechanism of anticancer Chinese medicine. However, this study only briefly verified the mechanism of one of the active 
ingredients in Pulsatilla. Therefore, further experiments are needed to verify the results of this study and to explore the mechanism of Pulsatilla as a potential anti-breast cancer drug.

\section{Declarations}

\section{Conflicts of interest}

The authors declare that they have no known competing financial interests or personal relationships that could have appeared to influence the work reported in this paper.

\section{References}

1. Sung H, Ferlay J, Siegel RL, Laversanne M, Soerjomataram I, Jemal A, Bray F (2020) Global Cancer Statistics 2020: GLOBOCAN Estimates of Incidence and Mortality Worldwide for 36 Cancers in 185 Countries. CA Cancer J Clin. https://doi.org/10.3322/caac.21660

2. Browne BC, O'Brien N, Duffy MJ, Crown J, O'Donovan N (2009) HER-2 signaling and inhibition in breast cancer. Curr Cancer Drug Targets 9(3):419-438. Epub 2009/05/16. doi: 10.2174/156800909788166484. PubMed PMID: 19442060

3. Vu T, Claret FX (2012) Trastuzumab: updated mechanisms of action and resistance in breast cancer. Frontiers in oncology 2:62. doi:10.3389/fonc.2012.00062. PubMed PMID: 22720269; PubMed Central PMCID: PMCPMC3376449. Epub 2012/06/22.

4. Bianchini G, Balko JM, Mayer IA, Sanders ME, Gianni L (2016) Triple-negative breast cancer: challenges and opportunities of a heterogeneous disease. Nature reviews Clinical oncology 13(11):674-690. doi:10.1038/nrclinonc.2016.66. PubMed PMID: 27184417; PubMed Central PMCID: PMCPMC5461122. Epub 2016/10/19.

5. Carey L, Winer E, Viale G, Cameron D, Gianni L (2010) Triple-negative breast cancer: disease entity or title of convenience? Nature reviews Clinical oncology 7(12):683-692.

doi:10.1038/nrclinonc.2010.154. PubMed PMID: 20877296. Epub 2010/09/30.

6. Harvey AL, Edrada-Ebel R, Quinn RJ (2015) The re-emergence of natural products for drug discovery in the genomics era. Nature reviews Drug discovery 14(2):111-129. doi:10.1038/nrd4510. PubMed PMID: 25614221. Epub 2015/01/24.

7. Newman DJ, Cragg GM (2016) Natural Products as Sources of New Drugs from 1981 to 2014. J Nat Prod 79(3):629-661. doi:10.1021/acs.jnatprod.5b01055. PubMed PMID: 26852623. Epub 2016/02/09.

8. Xu L, Cheng G, Lu Y, Wang S (2017) An active molecule from Pulsatilla chinensis, Pulsatilla saponin A, induces apoptosis and inhibits tumor growth of human colon cancer cells without or with 5-FU. Oncology letters 13(5):3799-3802. doi:10.3892/ol.2017.5884. PubMed PMID: 28529594; PubMed Central PMCID: PMCPMC5431750. Epub 2017/05/23. 
9. Guan Z, Chen L, Zhou Y, Luo Y, Cui Y, Liu R et al (2020) The synergistic antitumour effect of multicomponents from Pulsatilla chinensis saponins in $\mathrm{NCl}-\mathrm{H} 460$ lung cancer cell line through induction of apoptosis. Pharmaceutical biology 58(1):427-437 doi: 10.1080/13880209.2020.1761404. PubMed PMID: 32476531; PubMed Central PMCID: PMCPMC7337008

10. Liu Q, Chen W, Jiao Y, Hou J, Wu Q, Liu Y et al (2014) Pulsatilla saponin A, an active molecule from Pulsatilla chinensis, induces cancer cell death and inhibits tumor growth in mouse xenograft models. J Surg Res 188(2):387-395. doi:10.1016/j.jss.2014.01.026. PubMed PMID: 24576780. Epub 2014/03/01.

11. Liang Y, Xu X, Yu H, Li L, Hong T, Ji Q et al (2016) Raddeanoside R13 inhibits breast cancer cell proliferation, invasion, and metastasis. Tumour biology: the journal of the International Society for Oncodevelopmental Biology Medicine 37(7):9837-9847. doi:10.1007/s13277-015-4748-5. PubMed PMID: 26810189. Epub 2016/01/27.

12. Ru J, Li P, Wang J, Zhou W, Li B, Huang $C$ et al. TCMSP: a database of systems pharmacology for drug discovery from herbal medicines. J Cheminform. 2014;6:13. Epub 2014/04/17. doi:10.1186/1758-2946-6-13. PubMed PMID: 24735618; PubMed Central PMCID: PMCPMC4001360

13. Colozza M, Larsimont D, Piccart MJ (2005) Progesterone receptor testing: not the right time to be buried. Journal of clinical oncology: official journal of the American Society of Clinical Oncology 23(16):3867-3868. doi:10.1200/jco.2005.05.167. PubMed PMID: 15923594. author reply 9-70. Epub 2005/06/01.

14. Choudhary GS, Al-Harbi S, Almasan A. Caspase-3 activation is a critical determinant of genotoxic stress-induced apoptosis. Methods in molecular biology (Clifton, NJ). 2015;1219:1-9. Epub 2014/10/14. doi: 10.1007/978-1-4939-1661-0_1. PubMed PMID: 25308257

15. Hengartner MO. The biochemistry of apoptosis. Nature. 2000;407(6805):770-6. Epub 2000/10/26. doi: 10.1038/35037710. PubMed PMID: 11048727

16. Park C, Moon DO, Ryu CH, Choi B, Lee W, Kim GY et al. Beta-sitosterol sensitizes MDA-MB-231 cells to TRAIL-induced apoptosis. Acta Pharmacol Sin. 2008;29(3):341-8. Epub 2008/02/27. doi:

10.1111/j.1745-7254.2008.00761.x. PubMed PMID: 18298899

\section{Tables}

Due to technical limitations, table 1 is only available as a download in the Supplemental Files section.

\section{Figures}




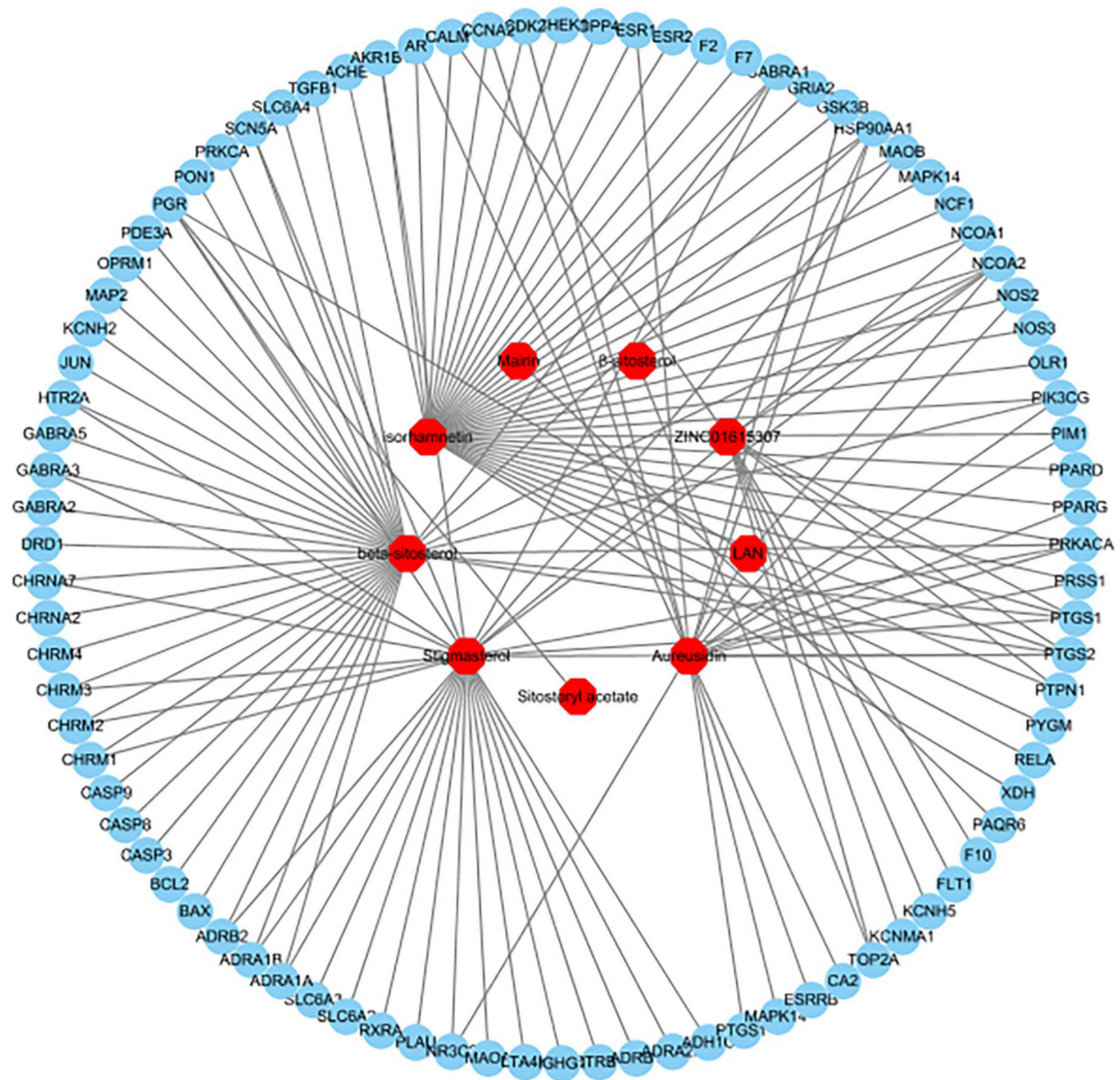

Figure 1

Compound-compound target network (red hexagon is the compound component, blue circle is the compound component target) 


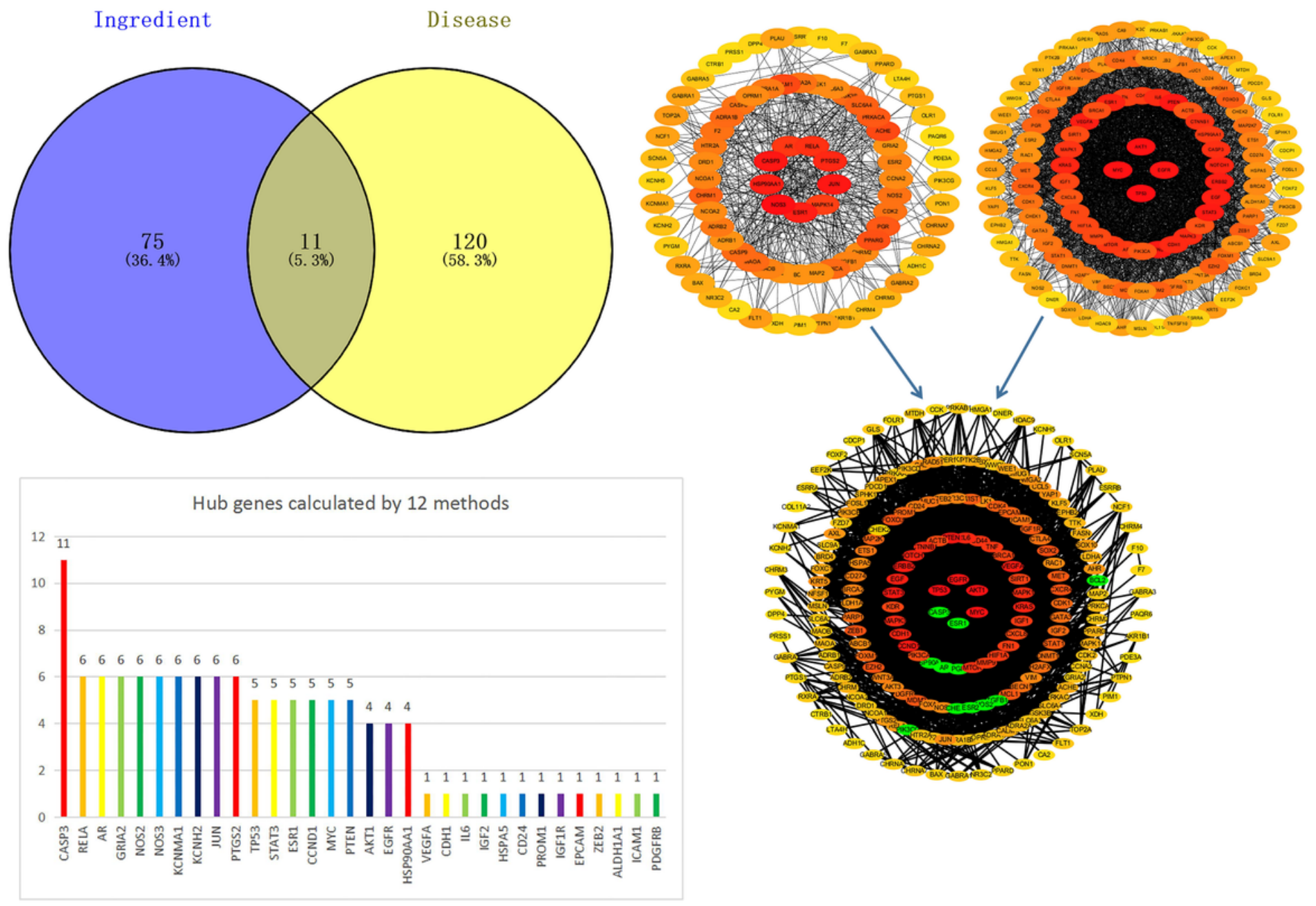

Figure 2

a. The intersection of compound component targets and triple negative breast cancer targets $b$.

Compound target-tri-negative breast cancer related target protein interaction network (marked in green is the intersection of compound target and triple-negative breast cancer target) c. Hub gene screening results calculated by 12 methods 

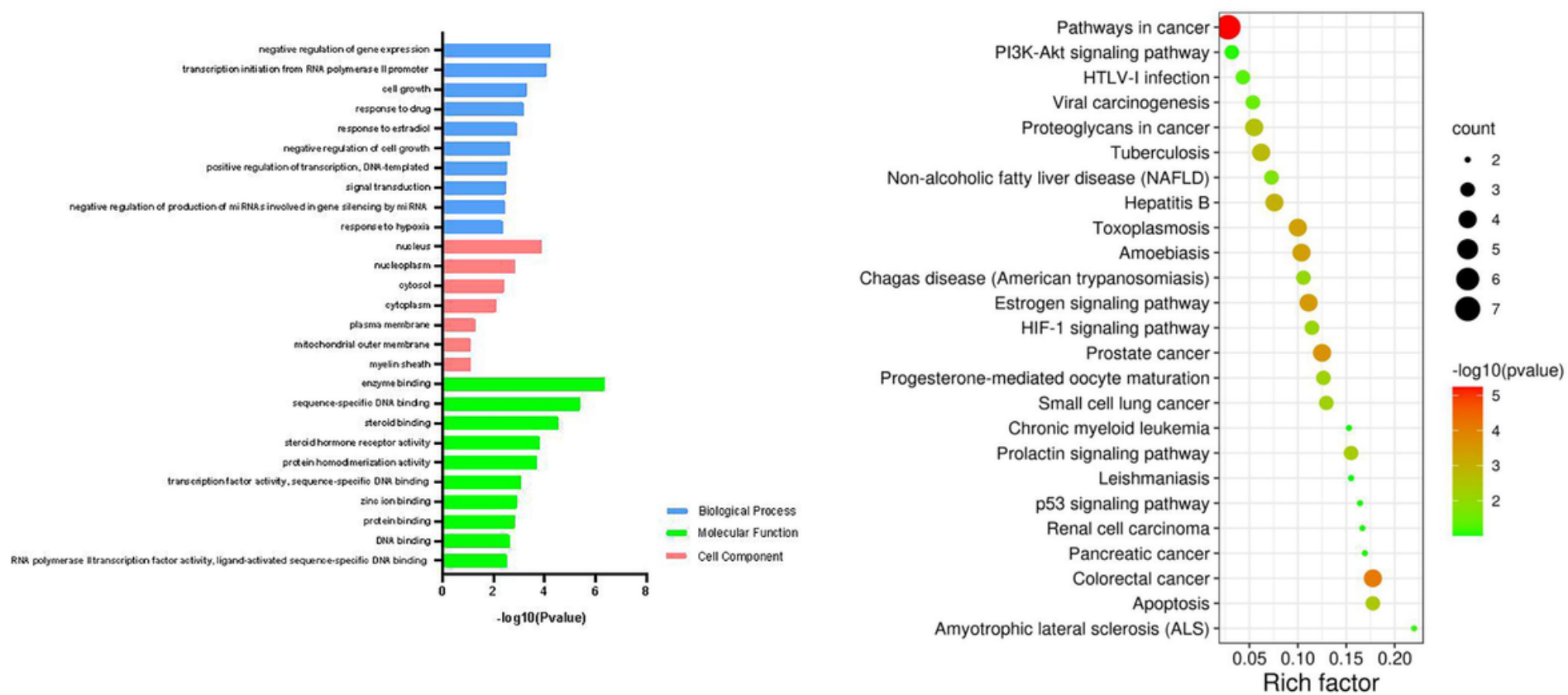

\section{Figure 3}

a GO enrichment map: the 10 most significant GO terms in the three GO categories. (The CC has only 7 entries) b Intersection gene enrichment analysis (vertical axis: KEGG pathway, horizontal axis: enrichment factor)

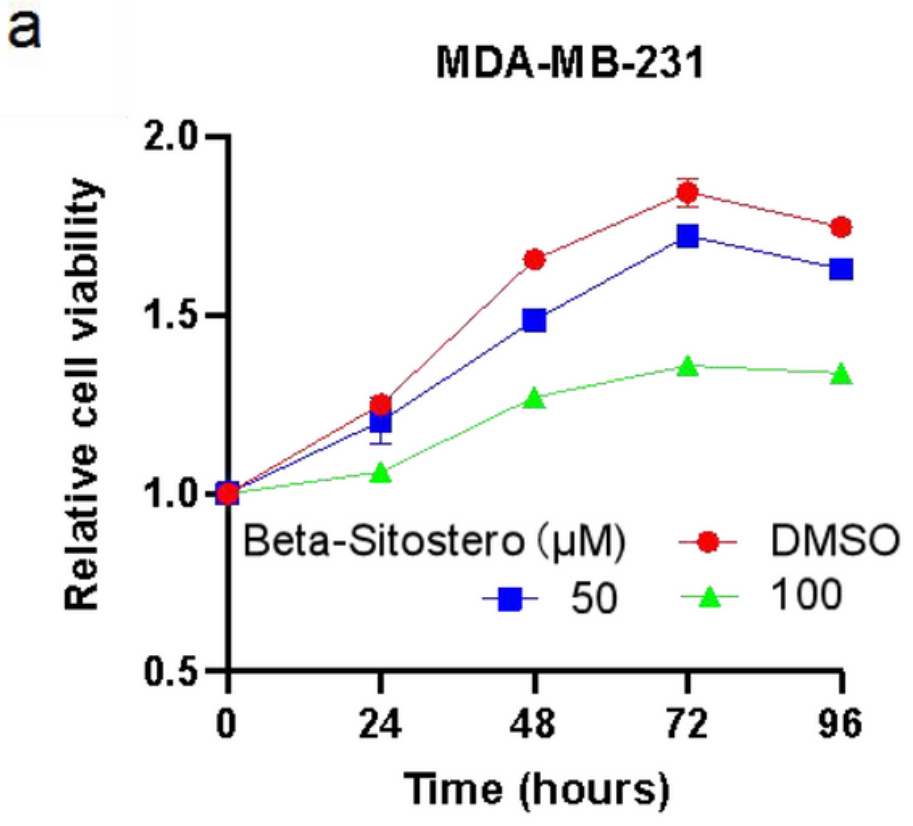

b

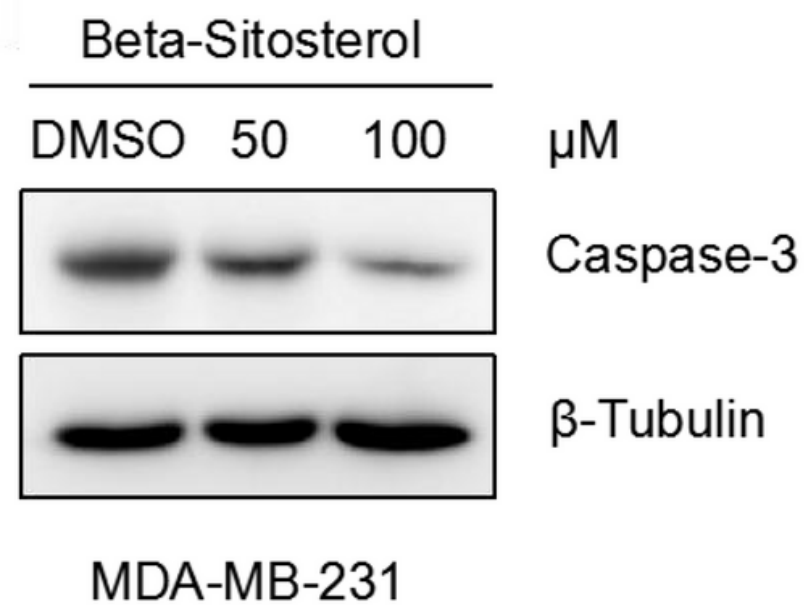

Figure 4

a. The proliferation curve of MDA-MB-231 cells treated with different concentrations of sitosterol b. Caspase-3 expression in MDA-MB-231 cells treated with different concentrations of sBeta-Sitosterol 


\section{Supplementary Files}

This is a list of supplementary files associated with this preprint. Click to download.

- Tables.docx 